\title{
Traumatic optic neuropathy-a contralateral finding: A case report
}

\author{
MARTIN KYNCL $^{1,2}$, JAN LESTAK $^{1,3}$, JAROSLAV TINTERA $^{1}$ and PAVEL HANINEC ${ }^{4}$ \\ ${ }^{1}$ JL Clinic, 15800 Prague; ${ }^{2}$ Department of Radiology, Second Faculty of Medicine, \\ Charles University in Prague and Motol University Hospital, 15006 Prague; ${ }^{3}$ Czech Technical University in Prague, \\ Faculty of Biomedical Engineering, $27201 \mathrm{Kladno} ;{ }^{4}$ Department of Neurosurgery, 3rd Faculty of Medicine, \\ Charles University in Prague, Teaching Hospital Královské Vinohrady, 10034 Prague, Czech Republic
}

Received October 4, 2018; Accepted February 28, 2019

DOI: $10.3892 /$ etm.2019.7445

\begin{abstract}
The present study demonstrates alterations of the contralateral side optic tract to an optic nerve traumatic lesion. Visual acuity of the right eye following Traumatic optic neuropathy (TON) remained at 0 following the injury. Electrophysiological examination using pattern electroretinogram revealed values reduced by $50 \%$ in the right eye compared with the left eye. Pattern visual-evoked potential evaluation indicated a bilateral lesion with a higher decrease following right eye stimulation. Magnetic resonance imaging revealed right optic nerve atrophy. Functional magnetic resonance imaging indicated decreased activity of the visual centre during left eye stimulation. The present study revealed contralateral visual tract alterations following unilateral injury, and hypothesize that the ganglion cells of the retina respond initially to glial activation. These changes are, in our view, followed by changes in the visual pathway.
\end{abstract}

\section{Introduction}

Traumatic optic neuropathy (TON) is an acute injury to the optic nerve due to trauma. Optic nerve axons may be affected directly or indirectly and cause partial or complete vision loss. Indirect trauma can occur after head trauma with intraneural edema, shearing of the optic nerve fibers, and altered cerebrospinal fluid circulation, which indirectly disrupts the function and anatomy of the optic nerve. The most common site of indirect TON is the part of the optic nerve that lies within the optic canal. The mechanism of TON can be mechanical shearing of optic nerve axons, ischemia, or apoptosis of adjacent neurons.

Experimental unilateral transection of the optic nerve also causes acute glial activation of the retina on the contralateral side (1-3). These experiments suggest that the processes of bilateral glial activation actually may also occur on the level

Correspondence to: Professor Jan Lestak, JL Clinic, V Hurkach 1296/10, 15800 Prague, Czech Republic

E-mail: lestak@seznam.cz

Key words: traumatic lesion of the optic nerve, electrophysiological examination, ganglion cell complex, magnetic resonance imaging of the retinal ganglion cells in the visual tract in humans. The current literature contains no mention of this topic, and we decided to present the case report below.

\section{Materials and methods}

A young man (born in 1981) suffered an injury to the right orbit upon hitting a concrete pole in the summer of 2013 while riding his bicycle. A severe vision disorder persisted after neurosurgical treatment with plastic surgery and reconstruction of the right orbit. The patient underwent his first complex assessment at our department six months after the injury. Standard ophthalmological tests and visual field testing (Medmont M700; Medmont International Pty Ltd., Nunawading, Australia) were performed on this patient after TON. The retinal nerve fibre layers (RNFL) and the ganglion cell complex (GCC) were tested using spectral domain optical coherence tomography (SD-OCT) RTVue-100. The pattern electroretinogram (PERG) and pattern visual evoked potential (PVEP; Roland Consult, Brandenburg an der Have, Germany) were performed using the ISCEV methodology. The stimulating board size was $30 \times 38 \mathrm{~cm}$ for both the PERG and PVEP. Square size at PERG-48 min, PVEP $60 \mathrm{~min}$. The distance of the stimulating board from the eye was $30 \mathrm{~cm}$, and the eye was set for this distance if necessary. Reversal rate was $4.02 \mathrm{~Hz}$ for PERG and $1.827 \mathrm{~Hz}$ for PVEP. The PERG voltage was detected using corneal DTL electrodes. Dish-shaped skin electrodes were placed $1 \mathrm{~cm}$ laterally from the outer corner of the eye. We evaluated the P50 and N95 latencies (ms) and the P50-N95 amplitudes (uV). In the PVEP (Fz-Oz electrodes), we evaluated the P100 latency and the N70-P100 amplitudes (uV).

Magnetic resonance imaging was used for structural and functional brain imaging. Magnetic resonance imaging (MRI) examinations were performed using the Philips Achieva 3T TX MR system (Philips Healthcare, Eindhoven, The Netherlands), with a magnetic field strength of 3 Tesla. Functional MRI (fMRI) uses blood oxygen level-dependent (BOLD) contrast. A standard 32-channel head coil was used, and each measurement was performed with a gradient-echo echo-planar imaging sequence $(\mathrm{TR} / \mathrm{TE}=3000 / 30 \mathrm{~ms}$, spatial resolution of $2 \times 2 \times 2 \mathrm{~mm}^{3}$ ). Optical stimulation was performed using a black/white checkerboard alternated with its negative image at a frequency of $2 \mathrm{~Hz}$. The visual size of the black and white checkerboard was $25.8 \times 6.2$ degrees. Measurements 
consisted of a sequence of five 30 -second active phase periods and five rest periods of the same length (for each of the ten dynamic scans). During the resting phase, a static crosshair situated in the centre of the visible field was projected for view fixation. Each measurement included 100 dynamic scans and took $5 \mathrm{~min}$. Each eye was examined using the means of separate fMRI measurements (LE and RE), and one control measurement was performed via stimulation of both eyes together (LE+RE).

Structural magnetic resonance imaging was performed in the basic planes using the following sequences: T1 TFE SAG 3D $1 \times 1 \times 1 \mathrm{~mm}^{3}, \mathrm{TR}=8.1 \mathrm{~ms}, \mathrm{TE}=3.7 \mathrm{~ms}$, NSA 1,170 slices; T2 mDIXON COR $2.5 \mathrm{~mm}, \mathrm{TR}=3,000 \mathrm{~ms}, \mathrm{TE}=80 \mathrm{~ms}$, 36 slices; T2 $4 \mathrm{~mm}$ TRA, TR=3,000 ms, TE=80 ms, NSA 1, 30 slices; FLAIR $4 \mathrm{~mm}, \mathrm{TR}=11,000 \mathrm{~ms}$, TE $=125 \mathrm{~ms}$; VenBold, TR=15 ms, TE=21 ms, NSA 2, 3DFFE 1x1 $1 \mathrm{~mm}^{3}$, 290 slices; T1 IR COR 5 mm, TR=3873 ms, TE=15 ms, NSA 1, 28 slices, gap 1; and DWI $4 \mathrm{~mm}, \mathrm{TR}=3,443 \mathrm{~ms}, \mathrm{TE}=76 \mathrm{~ms}$, b-factor 0-800, 30 slices. RNFL and GCC were examined using SD-OCT RTvue 100 with navigation on the right and fixation of a light point 37 and 50 months from the first ophthalmological testing on the left. PERG and PVEP were assessed on the first day of testing in February 2014.

Functional magnetic resonance imaging was performed nine months after the first ophthalmological testing.

\section{Results}

Enophthalmos was detected on the right, with the bulb in the 5-degree divergent position with free motility. Right-sided anisocoria was found with an amaurotic pupil, which responded to an indirect stimulus. Simple atrophy of the optic disc was also noted, but other ophthalmological findings were normal. This finding was normal on the left, and the pupil responded only to a direct light stimulus. Visual acuity was 0 and 1.0 (natural) on the right and left, respectively. Intraocular pressure (IOP) was $9 / 9 \mathrm{mmHg}$ (Fig. 1). Colour perception was unimpaired on the left. The visual field exhibited no decrease in sensitivity on the left.

The findings were identical at the given time points. A considerable decrease in RNFL and GCC thickness was observed on the right, and both of these findings were normal on the left (Fig. 2).

A considerable decrease in amplitudes in PERG was observed after stimulation of the right eye, up to $50 \%$ compared to the left eye. Values on the right were at approximately the lower limit of normal. The repeated examination approximately eight months later revealed a decrease in amplitude in the left eye, and this finding remained virtually unchanged until 37 months from the first examination. The amplitude values increased at this time. The measured amplitude values remained virtually unchanged on the right throughout the follow-up period. The last examination was completed 52 months after the first examination and revealed a considerable improvement in amplitudes in the left eye (Fig. 3). The amplitude values of the PVEP were markedly pathological on both sides from the first examination and exhibited a more profound decrease after stimulation of the right eye. An increase in PVEP amplitudes was observed after stimulation of the left eye starting from the 37-month time

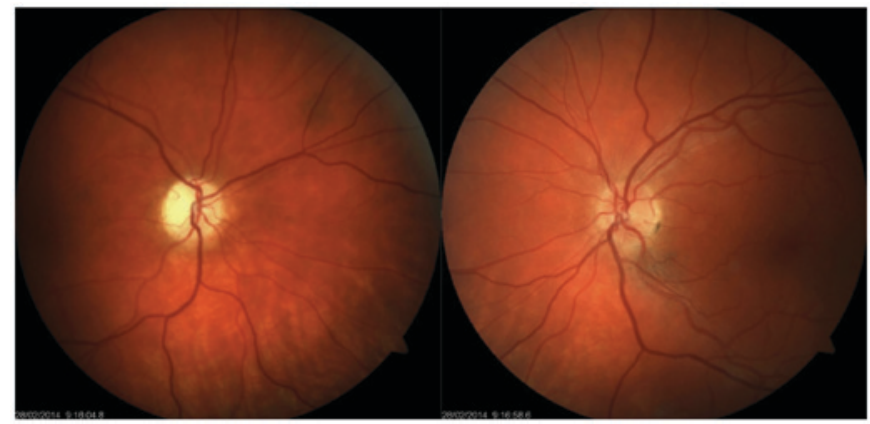

Figure 1. Colour photograph of the eye fundus. Simple atrophy of the optic disc on the right; more numerous glial cells around larger vascular bundles on the left.

point. The values were considerably abnormal despite this increase (Fig. 4).

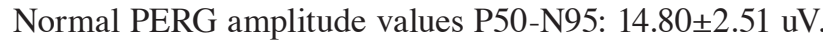
Normal PVEP amplitude values N70-P100: 12.22 $\pm 3.22 \mathrm{uV}$ (4).

Functional magnetic resonance imaging was performed nine months after the first ophthalmological testing. fMRI exhibited a considerable decrease in visual cortex activity after stimulation of the left eye. A further decrease was observed ten months after the first ophthalmological testing, followed by culmination with a gradual increase in voxel activity, similarly as the PVEP. Values remained below the normal range despite this increase. fMRI activity of the right eye alone was not examined because its fixation to a relatively small stimulation area could not be ensured. The following mean values were observed in a healthy population using our methodology: fMRI response was compared with normal control database which is $9,200 \pm 2,700$ monocular voxels for the corresponding age group (Fig. 5).

Structural MRI revealed post-contusion alternations of the brain in the frontal-basal and frontal-polar regions on the right, with minor haemosiderin deposits. No other foci were observed, and no acute cerebral ischaemia was demonstrated. Periventricular atrophy of the frontal horn of the right lateral ventricle and an atrophying process of the entire optic nerve on the right and the right half of the optic chiasm were found (Fig. 6).

\section{Discussion}

Glial activation in the retina on the ipsilateral and contralateral sides was demonstrated in experimental models in the literature (1-3). These experimental conclusions suggest that the processes of bilateral glial activation may also occur on the level of the retinal ganglion cells themselves and the visual tract in humans. This hypothesis was supported by our electrophysiological testing of other patients after TON. The normal visual field and colour perception of our patients revealed no abnormalities on the contralateral side. This result was likely because an up to $25-30 \%$ loss of retinal ganglion cells is needed to detect the first perimetric changes using static automatic perimetry. Colour perception tends to be affected similarly.

RNFL and GCC examinations detected a loss only on the ipsilateral side. These alternations may also be found on the contralateral side, but they cannot be captured using current 
OD

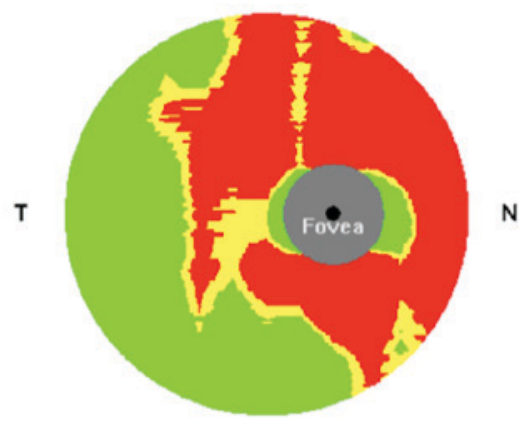

Optic Nerve Head Map

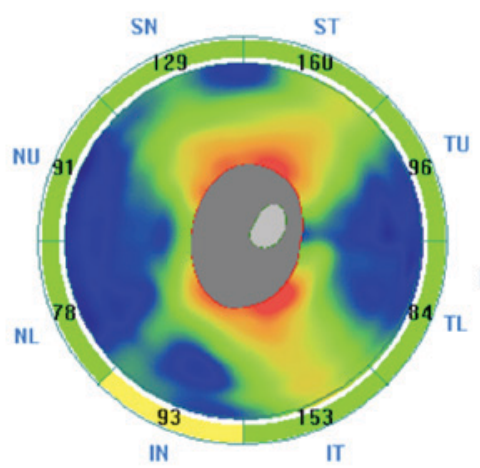

IN

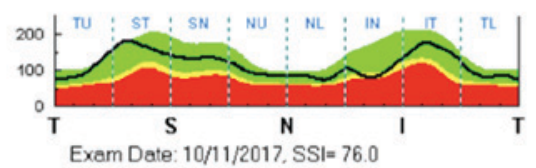

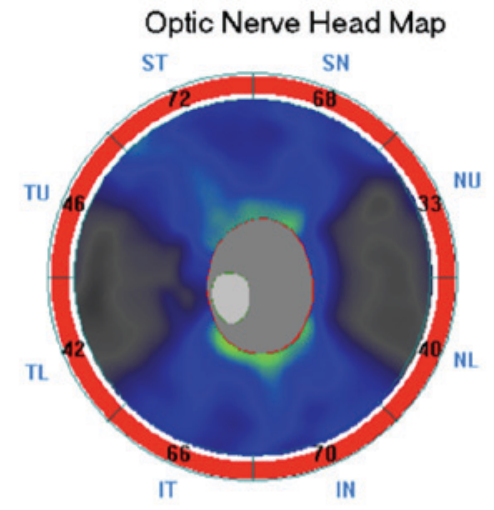

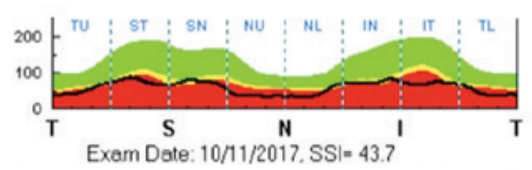

GCC Significance

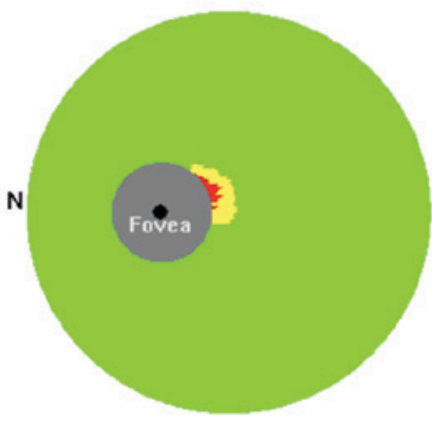

Exam Date: $10 / 11 / 2017, \mathrm{SS} \mid=66.5$

\begin{tabular}{|l|l|l|}
\hline RNFL. Parameters & OD & \multicolumn{1}{l|}{ OS } \\
\hline \multicolumn{1}{|l|}{ Avg. RNFL } & 54.57 & 110.55 \\
\hline Sup. Avg & 54.78 & 118.96 \\
\hline Inf. Avg & 54.35 & 102.14 \\
\hline & & \\
\hline
\end{tabular}

$D>5 \%$ Within Normal P $<5 \%$ Borderine

\begin{tabular}{|c|c|c|}
\hline GCC Parameters & $\infty$ & os \\
\hline Avg. $G C C(\mu \mathrm{m})$ & 72.45 & 103.91 \\
\hline Sup. $G C C(\mu m)$ & 69.05 & 104.45 \\
\hline Inf. $G C C(\mu \mathrm{m})$ & 75.82 & 103.37 \\
\hline FLV (\%) & 11.646 & 0.815 \\
\hline $\operatorname{GV}(\%)$ & 22.774 & 0.961 \\
\hline
\end{tabular}

T

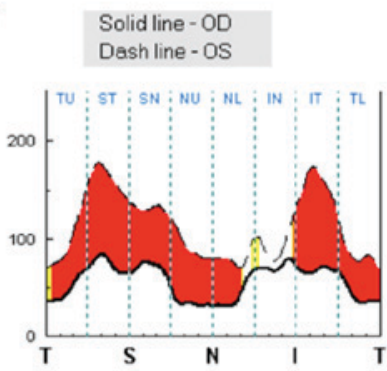

Figure 2. The RNFL and the GCC. RNFL, retinal nerve fibre layer; GCC, ganglion cell complex.

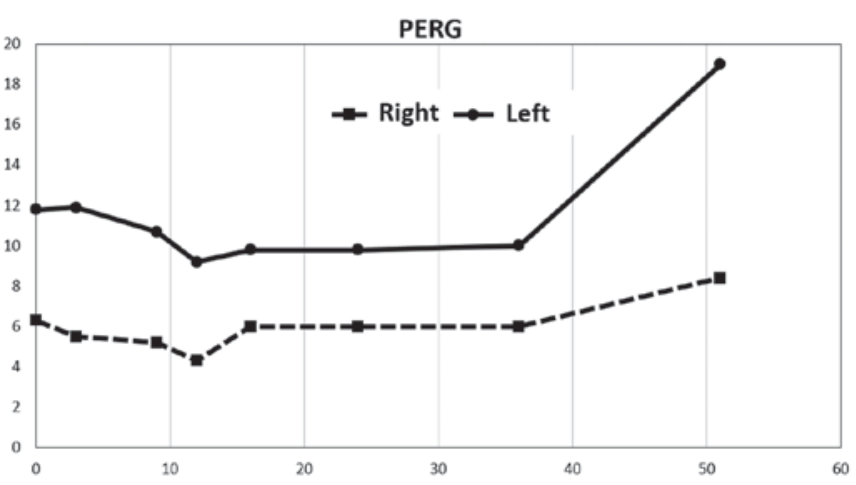

Figure 3. Development of PERG amplitudes (P50-N95) over time. N95 latency was $100 \mathrm{~ms}$ starting from the first measurement and revealed no changes in the right eye. A constant value of $95 \mathrm{~ms}$ was measured in the left eye. PERG, pattern electroretinogram.

methods. OCT only scans GCC thickness, which may be affected by glial proliferation.

The problem of damage to the contralateral optic nerve, or more precisely the contralateral visual tract, after TON in human medicine, is not known from the literature.
Liu et al (5) discovered PERG changes in the ipsilateral and contralateral eyes after unilateral damage to the optic nerve in a murine model. These findings preceded morphological alterations in the layer of retinal ganglion cells (5). The PERG findings obtained in our patient confirm this finding. However, we cannot explain the increased amplitude on the damaged side and the contralateral side 43 months after the injury. PVEP amplitudes also increased, approximately from 30 months after the injury. Ganglion cells in the retina pass through the pathological process of shrinkage before cell death. This corresponds to a decrease in their action potential, and this change can be diagnosed with PERG, even when OCT findings of the ganglion cell complex are normal.

We also considered the possibility of influencing PVEP associated with brain contusions and contralateral side optic tract changes. The authors have their own experience with contralateral side optic tract injuries related to TON lesions of the optic nerve caused by tumor compression.

No PVEP findings are available in the literature for human TON, and no fMRI examinations are found. Our results indicate damage to the entire visual tract that was not limited to the 
PVEP

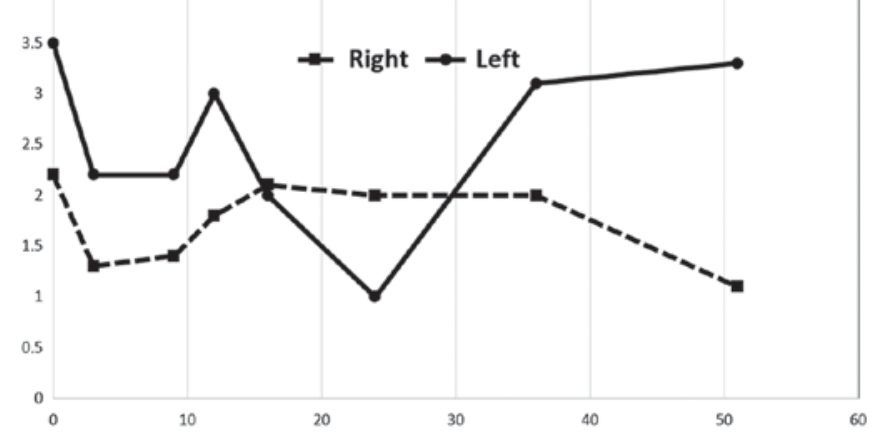

Figure 4. Development of PVEP amplitudes (N75-P100) over time. P100 latency remained unchanged throughout the follow-up period. PVEP, pattern visual evoked potential.

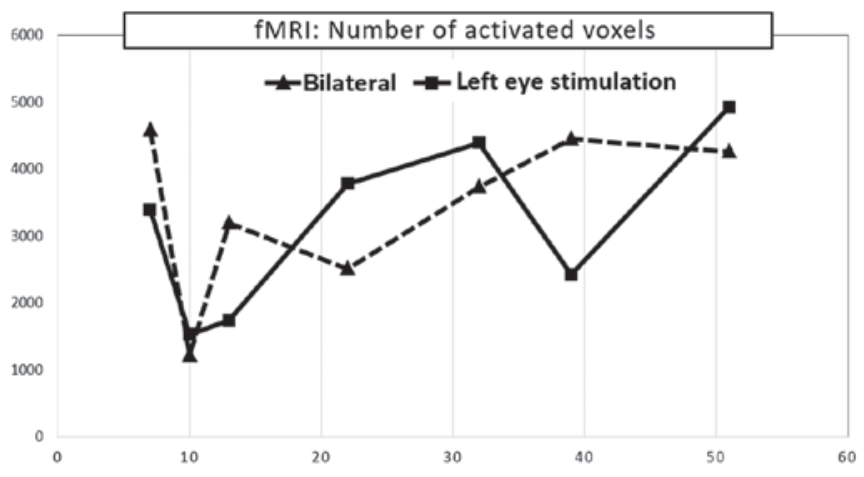

Figure 5. Development of fMRI after stimulation of the left eye and both eyes over time. fMRI, functional MRI; MRI, magnetic resonance imaging.

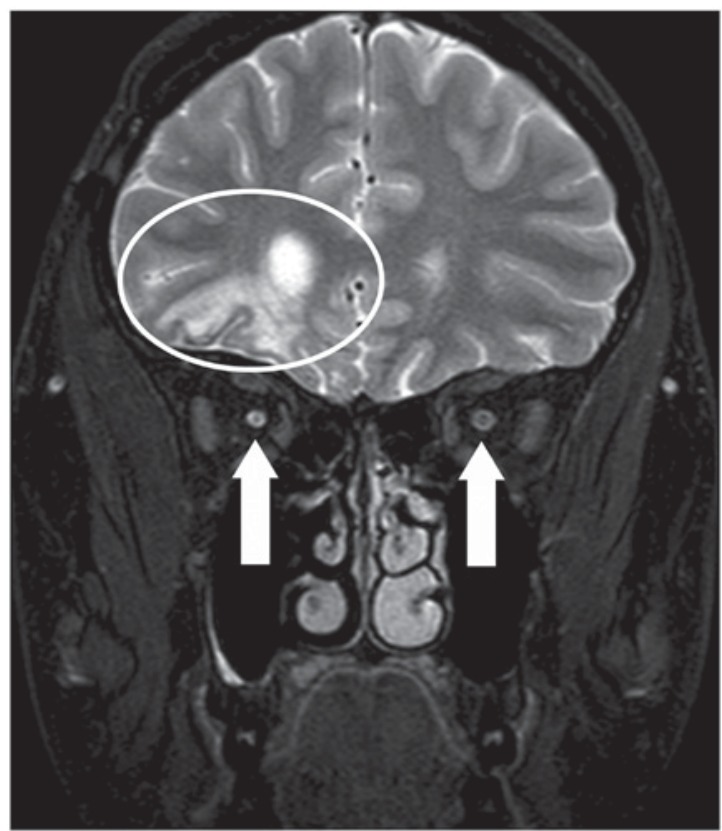

Figure 6. MRI scan (coronal plane, T2 mDIXON) with suppressed signal from fat. Post-contusion alterations in the right hemisphere (the ellipse). The arrows indicate the sizes of the optic nerves, with a smaller diameter on the right. MRI, magnetic resonance imaging.

damaged side but also included the contralateral side. We are convinced that the ganglion cells of the retina respond initially to glial activation. These changes are, in our view, followed by changes in the visual pathway.

Potential therapy should be briefly mentioned. In Czech literature, the first solid opinion on this topic was adopted by Otradovec (6), who summarized his TON results in ten points: i) Injury to one optic nerve is the most common visual tract disorder after a blunt injury of the head; ii) the vision disorder develops at the moment of the injury; iii) the condition is characterized by a remarkable capability of spontaneous restitution of the visual functions, which may be expected in approximately one-half of injured persons. The first signs of improvement occur 3 to 4 days after injury and reach peak intensity in the first 3-4 weeks; iv) altitudinal scotoma is a typical perimetric defect; v) the bulb is generally not damaged, and pupil function disorder is the only objective sign of the sensory disorder; vi) cranial bone fractures are common, but not obligatory, finding; vii) surgical revisions of 17 optic nerves in 16 patients with post-traumatic amaurosis never revealed any changes that could be rectified by the procedure; viii) sagittal displacement of the brain inside the skull at the moment of impact is likely involved; ix) indications for surgical revision of the canalicular region in cases of post-traumatic amaurosis are exceptional, and generally, they are extraocular, such as liquorrhoea, pneumocephalus, and similar reasons; $x$ ) the first four days after the injury are a contraindication for the surgical procedure, i.e., the period when spontaneous restitution of visual functions may begin.

We have previous experience with this type of problem. We previously described the case of a 22-year-old male with a car accident injury and 0 visual acuity on the left. The optic canal was decompressed on the second day after injury, and the patient's visual acuity began to improve as early as the second postoperative day, as seen by his ability to recognize light from dark. The patient's visual acuity improved to 0.1 one month after injury. These visual function results are consistent with the above-mentioned evaluations of Otradovec (7).

Review papers in recent years have not adopted a uniform position for this problem. No evidence of TON therapy exists. Therefore, there is insufficient consensus among clinicians for the suitability of TON therapy. Our overview of recent literature revealed no reliable evidence that suggests that TON therapy using corticosteroids, surgical decompression of the optic canal, or both provides any benefit compared to mere observation. Furthermore, these interventions pose an additional risk that may not warrant their routine use $(8,9)$.

Our study demonstrated that unilateral damage to the optic nerve in TON was also associated with considerable alterations of the contralateral visual tract, including functional alterations of the retina.

\section{Acknowledgements}

Not applicable.

\section{Funding}

The present study was supported by MH CZ-DRO, Motol University Hospital, Prague, Czech Republic (grant no. 00064203). Progres Q35. The present study was supported by the Charles University research program PROGRES Q35. 
The present study was supported by the EU Structural Funds OPP competitiveness (grant no. CZ.2.16/3.1.00/21532).

\section{Availability of data and materials}

The datasets used and/or analyzed during the current study are available from the corresponding author on reasonable request.

\section{Author's contributions}

All the authors were involved in conceiving and designing the study. KM and LJ drafted and wrote the manuscript. KM, LJ and TJ were responsible for the collection and analysis of the patient data. HP critically revised the manuscript for important intellectual content. All authors read and approved the final version of the manuscript.

\section{Ethics approval and consent to participate}

The study protocol was approved by the local Ethics Committee of JL Clinic, Faculty of Biomedical Engineering, Czech Technical University in Prague, and the study was performed in accordance with Good Clinical Practice and the Declaration of Helsinki. The patient provided written informed consent.

\section{Patient consent for publication}

The patient provided written informed consent; however, the authors made efforts to remove the identifying information to protect the privacy of the patient.

\section{Disclosure of interest}

The authors declare that they have no competing interests.

\section{References}

1. Bodeutsch N, Siebert H, Dermon C and Thanos S: Unilateral injury to the adult rat optic nerve causes multiple cellular responses in the contralateral site. J Neurobiol 38: 116-128, 1999.

2. Panagis L, Thanos S, Fischer D and Dermon CR: Unilateral optic nerve crush induces bilateral retinal glial cell proliferation. Eur J Neurosci 21: 2305-2309, 2005.

3. Cen LP, Han M, Zhou L, Tan L, Liang JJ, Pang CP and Zhang M: Bilateral retinal microglial response to unilateral optic nerve transection in rats. Neuroscience 311: 56-66, 2015.

4. Lestak J, Nutterova E, Pitrova S, Krejcova H, Bartosova L and Forgacova V: High tension versus normal tension glaucoma. A comparison of structural and functional examinations. J Clin Exp Ophthalmol 3: 1-4, 2012.

5. Liu Y, McDowell CM, Zhang Z, Tebow HE, Wordinger RJ and Clark AF: Monitoring retinal morphologic and functional changes in mice following optic nerve crush. Invest Ophthalmol Vis Sci 55: 3766-3774, 2014.

6. Otradovec J: Klinická Neurooftalmologie. Prague Grada Publishing, 2003.

7. Haninec P, Klener J, Houst'ava L and Lesták J: Restoration of vision after surgical treatment of traumatic optic neuropathy of an uncommon type. Case report and literature review. Cesk Slov Oftalmol 52: 249-252, 1996 (In Czech).

8. Kumaran AM, Sundar G and Chye LT: Traumatic optic neuropathy: A review. Craniomaxillofac Trauma Reconstr 8: 31-41, 2015.

9. Chaon BC and Lee MS: Is there treatment for traumatic optic neuropathy? Curr Opin Ophthalmol 26: 445-449, 2015. 